\title{
Calculations of light scattering from isolated and interacting metallic nanowires of arbitrary cross section by means of Green's theorem surface integral equations in parametric form
}

\author{
Vincenzo Giannini* and Jose A. Sánchez-Gil \\ Instituto de Estructura de la Materia, Consejo Superior de Investigaciones Científicas, \\ Serrano 121, 28006 Madrid, Spain \\ *Corresponding author: vingenzino@iem.cfmac.csic.es
}

Received February 6, 2007; revised May 11, 2007; accepted May 17, 2007; posted May 24, 2007 (Doc. ID 79858); published August 9, 2007

\begin{abstract}
We study theoretically the light scattering from metal wires of arbitrary cross section, with emphasis on the occurrence of plasmon resonances. We make use of the rigorous formulation of the Green's theorem surface integral equations of the electromagnetic wave scattering, written for an arbitrary number of scatterers described in parametric form. We have investigated the scattering cross sections for nanowires of various shapes (circle, triangles, rectangles, and stars), either isolated or interacting. The relationship between the cross sectional shape and the spectral dependence of the plasmon resonances is studied, including the impact of nanoparticle coupling in the case of interacting scatterers. Near-field intensity maps are also shown that shed light on the plasmon resonance features and the occurrence of local field enhancements. () 2007 Optical Society of America

OCIS codes: $290.5850,240.6680$.
\end{abstract}

\section{INTRODUCTION}

Recently, the development of nanofabrication techniques, together with modern nanocharacterization techniques such as dark-field and near-field optical microscopies, has led to a new interest in processes concerning localization and guiding of electromagnetic (EM) waves in metal nanostructures [1-7]. Such techniques hold promise of potential applications in imaging, surface-enhanced Raman spectroscopy (SERS), lithography, optoelectronic devices, and biosensing. In recent years, the increasing research effort in the field of plasmonics-the study of EM field confinement and enhancement via surface plasmons (SPs)—has prompted advances in the theoretical studies of light scattering from nanostructures [8-17]. One of the most important applications of localized plasmons in nanoparticles is SERS [9,18-20]; a very high sensitivity has been achieved with this spectroscopic technique, making possible, in fact, single-molecule detection [21,22].

Light scattering from small particles has been studied since long ago (see [23] and references therein). Analytical solutions for the EM fields are known only for particles of a very simple shape, such as cylinders, spheres, or ellipsoids [23]. When we are interested in interacting particles of arbitrary shape, numerical calculations are required. Different formulations exist that rigorously describe the response of metallic nanoparticles of arbitrary shape: finite-difference time domain [24], dyadic Green's function technique $[9,25]$, and various implementations of the boundary integral methods [8,26-28] based on Green's second theorem (when the dielectric function is constant within the nanoparticle volume) are the most widely employed.

In this work we calculate the light scattering by twodimensional particles of arbitrary shape. The particles studied might exhibit corners and tips, and can be interacting; in some cases, extremely large local enhancements might take place. Calculations are based on the Green's theorem surface integral equation formulation $[26,27,29,30]$, herein modified to deal with surface profiles in parametric form. With this method, we show that metal nanowires with a nonregular cross section exhibit a complex resonance spectrum; in turn we study the nearfield and charge distributions at resonant frequencies, where a large scattering cross section is found.

The work is organized as follows. In Section 2 we present the scattering equations obtained by means of the Green's theorem surface integral equation formulation in parametric form. Next we show the high versatility of this formulation, including results for triangular, cylindrical, rectangular, and star-shape nanoparticles, either isolated (Section 3) or interacting (Section 4). We draw conclusions from the results in Section 5 .

\section{THEORETICAL FORMULATION}

A. Surface Integral Equations in Parametric Form We study the light scattered by two-dimensional particles, i.e., particles having a translational symmetry along one direction, such as wires or infinitely long cylin 


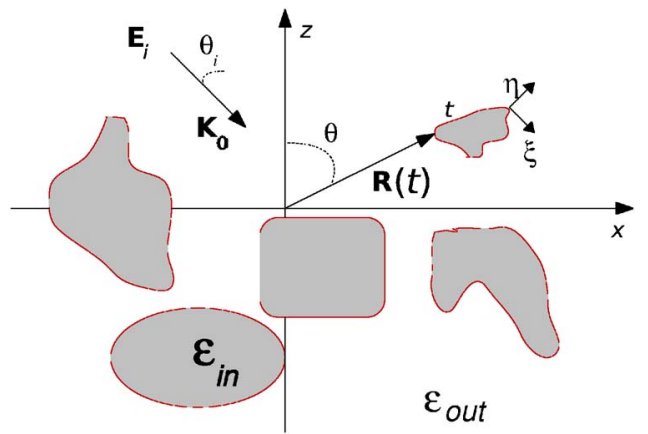

Fig. 1. (Color online) Schematic diagram of the scattering geometry.

ders. The physical system we consider is shown in Fig. 1. The particles are illuminated in the plane of the figures with a linearly polarized, monochromatic plane electromagnetic wave of frequency $\omega$ at an angle $\theta_{0}$ with the $z$ axis. The polarization is defined as follows: the magnetic (electric) field is perpendicular to the $x z$ plane for $p(s)$ polarization, also known as transverse magnetic (transverse electric). The restriction to two-dimensional systems has the advantage that it notably simplifies the formulation; in fact, we can reduce the initial three-dimensional vectorial problem to a two-dimensional scalar one, where the EM field is entirely described by the $y$ component of, respectively, the electric field for $s$ polarization $E_{y}^{(s)}(\mathbf{r})$ $=E(\mathbf{r})$ and the magnetic field for $p$ polarization $H_{y}^{(p)}(\mathbf{r})$ $=H(\mathbf{r})$.

Recall that for $s$ polarization, surface plasmons (which are transverse magnetic) cannot be excited. It should be emphasized that this simplification stems only from the scattering geometry, so that the full vector EM character of the problem is preserved. The scattering configuration consists of $N$ objects (metal or dielectric) with arbitrary shapes described by an isotropic, homogeneous, frequency-dependent dielectric function $\varepsilon_{j}^{(i n)}(\omega)$ in a dielectric background characterized by a homogeneous, isotropic, frequency-dependent dielectric function $\varepsilon^{(\text {out })}(\omega)$. (The dielectric functions are taken from [31].) The surface profile $\Gamma_{j}$ of the $j$ th scatterer is represented by the continuous vector-valued function $\mathbf{R}_{j} \equiv\left(\xi_{j}, \eta_{j}\right)$, where $\left(\xi_{j}, \eta_{j}\right)$ are the coordinates of a point of the profile in the $x z$ plane. We describe surface profiles as parametric curves: $\mathbf{R}_{j}(t)$ $\equiv\left[\xi_{j}(t), \eta_{j}(t)\right]$.

\section{1. $p$ Polarization}

Let us focus first on the calculation of the scattered EM field in the case of $p$ polarization. The method of calculation is based on the integral equations resulting from the application of Green's second integral theorem (with Sommerfeld radiation condition) [26,27,30,32,33]. Employing Green's integral theorem outside the scatterers, the magnetic field for $p$ polarization can be written in parametric coordinates as

$$
\begin{gathered}
H^{(i)}(\mathbf{r})+\frac{1}{4 \pi} \sum_{j} \int_{\Gamma_{j}}\left\{H^{(\text {out })}\left[\mathbf{R}_{j}(t)\right] \frac{\partial G^{(\text {out })}\left[\mathbf{r}, \mathbf{R}_{j}(t)\right]}{\partial N_{j}}\right. \\
\left.-G^{(\text {out })}\left[\mathbf{r}, \mathbf{R}_{j}(t)\right] \frac{\partial H^{(\text {out })}\left(\mathbf{R}_{j}\right)}{\partial N_{j}}\right\} \mathrm{d} t=H^{(\text {out })}(\mathbf{r}),
\end{gathered}
$$

$$
=0, \quad \mathbf{r} \text { inside }
$$

where $H^{(i)}(\mathbf{r})$ is the incident magnetic field.

If the Green's theorem is applied inside the scatterers, one obtains the following two integral equations for each one $(j=1, \ldots N)$ :

$$
\begin{aligned}
-\frac{1}{4 \pi} \int_{\Gamma_{j}}\left\{H^{(i n)}\left[\mathbf{R}_{j}(t)\right] \frac{\partial G_{j}^{(i n)}\left[\mathbf{r}, \mathbf{R}_{j}(t)\right]}{\partial N_{j}}\right. & \\
\left.-G_{j}^{(i n)}\left[\mathbf{r}, \mathbf{R}_{j}(t)\right] \frac{\partial H^{(i n)}\left(\mathbf{R}_{j}\right)}{\partial N_{j}}\right\} \mathrm{d} t & =0, \quad \mathbf{r} \text { outside } \Gamma_{j}, \\
& =H^{(i n)}(\mathbf{r}), \quad \mathbf{r} \text { inside } \Gamma_{j} .
\end{aligned}
$$

The superscripts (out,in) denote the magnetic fields $H^{\text {(out,in) }}(\mathbf{r})$ evaluated, respectively, outside and inside the scatterers. In the above equations, the normal derivative (not normalized) is defined as

$$
\frac{\partial}{\partial N_{j}}=\left[-\eta_{j}^{\prime}(t) \frac{\partial}{\partial x}+\xi_{j}^{\prime}(t) \frac{\partial}{\partial z}\right],
$$

and the Green's functions $G^{(\text {out }, \text { in })}(\mathbf{r}, \mathbf{R})$ can be expressed in terms of the Hankel function of the first kind:

$$
\begin{aligned}
& G^{(\text {out })}(\mathbf{r}, \mathbf{R})=i \pi H_{0}^{(1)}\left[\frac{\omega}{c} \sqrt{\varepsilon^{(\text {out })}}|\mathbf{r}-\mathbf{R}|\right], \\
& G_{j}^{(i n)}(\mathbf{r}, \mathbf{R})=i \pi H_{0}^{(1)}\left[\frac{\omega}{c} \sqrt{\varepsilon_{j}^{(i n)}}|\mathbf{r}-\mathbf{R}|\right] .
\end{aligned}
$$

We now define $\mathcal{H}_{j}(t)$ and $\mathcal{L}_{j}(t)$ as the source functions (unknown) according to the continuity conditions across the interface of the $j$ th scatterer:

$$
\begin{gathered}
\mathcal{H}_{j}(t)=\left.H^{(\text {out })}(\mathbf{r})\right|_{\mathbf{r} \rightarrow R_{j}^{+}(t)}=\left.H^{(\text {in })}(\mathbf{r})\right|_{\mathbf{r} \rightarrow R_{j}^{-}(t)}, \\
\mathcal{L}_{j}(t)=\left[\frac{\partial H^{(\text {out })}(\mathbf{r})}{\partial N_{j}}\right]_{\mathbf{r} \rightarrow R_{j}^{+}(t)}=\frac{\varepsilon^{(\text {out })}}{\varepsilon_{j}^{(\text {in })}}\left[\frac{\partial H^{(i n)}(\mathbf{r})}{\partial N_{j}}\right]_{\mathbf{r} \rightarrow R_{j}^{-}(t)},
\end{gathered}
$$

where the superscripts $+(-)$ indicate that the limit is taken with $\mathbf{r}$ from outside (inside) the scatterers. By evaluating Eqs. (1a) and (2a) at the interfaces and invoking the continuity conditions (5) across them, we obtain a set of coupled integral equations:

$$
\begin{array}{r}
H^{(i)}\left(\mathbf{R}_{l}\right)+\frac{1}{4 \pi} \sum_{j} \int_{\Gamma_{j}}\left\{\mathcal{H}_{j}(t) \frac{\partial G^{(\text {out })}\left[\mathbf{R}_{l}, \mathbf{R}_{j}(t)\right]}{\partial N_{j}}\right. \\
\left.-G^{(\text {out })}\left[\mathbf{R}_{l}, \mathbf{R}_{j}(t)\right] \mathcal{L}_{j}(t)\right\} \mathrm{d} t=\mathcal{H}_{l}(t), \\
\quad l=1, \ldots, N ;
\end{array}
$$




$$
\begin{gathered}
\frac{1}{4 \pi} \int_{\Gamma_{j}}\left\{\mathcal{H}_{j}(t) \frac{\partial G^{(i n)}\left[\mathbf{R}_{l}, \mathbf{R}_{j}(t)\right]}{\partial N_{j}}-\frac{\varepsilon_{j}^{(i n)}(\omega)}{\varepsilon^{(o u t)}(\omega)}\right. \\
\left.G^{(i n)}\left[\mathbf{R}_{l}, \mathbf{R}_{j}(t)\right] \mathcal{L}_{j}(t)\right\} \mathrm{d} t=0, \\
\quad l, j=1, \ldots, N .
\end{gathered}
$$

These coupled integral equations are converted into matrix equations using a simple quadrature approximation to evaluate the integral over small intervals. The coupled system of matrix equations can then be solved numerically to determine the source functions $\mathcal{H}_{j}(t)$ and $\mathcal{L}_{j}(t)$ from Eqs. (6a) and (6b). Using these source functions, the near- and the far-field amplitudes can easily be determined. Note that the scattering problem scales with the surface of the particle and not with its volume, which is a decisive advantage in numerical simulations.

\section{2. s Polarization}

Likewise, the following integral equations are obtained by using the Green's integral theorem for the only nonzero component of the electric field in the case of $s$ polarization:

$$
\begin{aligned}
& E^{(i)}(\mathbf{r})+\frac{1}{4 \pi} \sum_{j} \int_{\Gamma_{j}}\left\{E^{(\text {out })}\left[\mathbf{R}_{j}(t)\right] \frac{\partial G^{(\text {out })}\left[\mathbf{r}, \mathbf{R}_{j}(t)\right]}{\partial N_{j}}\right. \\
& \left.-G^{(o u t)}\left[\mathbf{r}, \mathbf{R}_{j}(t)\right] \frac{\partial E^{(o u t)}\left(\mathbf{R}_{j}\right)}{\partial N_{j}}\right\} \mathrm{d} t=E^{(o u t)}(\mathbf{r}), \\
& \text { r outside, } \\
& =0, \quad \mathbf{r} \text { inside, } \\
& -\frac{1}{4 \pi} \int_{\Gamma_{j}}\left\{E^{(i n)}\left[\mathbf{R}_{j}(t)\right] \frac{\partial G_{j}^{(i n)}\left[\mathbf{r}, \mathbf{R}_{j}(t)\right]}{\partial N_{j}}\right. \\
& \left.-G_{j}^{(i n)}\left[\mathbf{r}, \mathbf{R}_{j}(t)\right] \frac{\partial E^{(i n)}\left(\mathbf{R}_{j}\right)}{\partial N_{j}}\right\} \mathrm{d} t=0, \quad \mathbf{r} \text { outside } \Gamma_{j}, \\
& =E^{(i n)}(\mathbf{r}), \quad r \text { inside } \Gamma_{j} .
\end{aligned}
$$

We define the (unknown) source functions $\mathcal{E}_{j}(t)$ and $\mathcal{F}_{j}(t)$ as

$$
\begin{gathered}
\mathcal{E}_{j}(t)=\left.E^{(\text {out })}(\mathbf{r})\right|_{\mathbf{r} \rightarrow R_{j}^{+}(t)}=\left.E^{(\text {in })}(\mathbf{r})\right|_{\mathbf{r} \rightarrow R_{j}^{-}(t)}, \\
\mathcal{F}_{j}(t)=\left[\frac{\partial E^{(\text {out })}(\mathbf{r})}{\partial N_{j}}\right]_{\mathbf{r} \rightarrow R_{j}^{+}(t)}=\left[\frac{\partial E^{(\text {in })}(\mathbf{r})}{\partial N_{j}}\right]_{\mathbf{r} \rightarrow R_{j}^{-}(t)},
\end{gathered}
$$

where the continuity conditions have been explicitly accounted for. To solve for the source functions, we evaluate Eqs. (7a) and (8a) at the interfaces, thus obtaining the following system of coupled integral equations:

$$
\begin{gathered}
E^{(i)}\left(\mathbf{R}_{l}\right)+\frac{1}{4 \pi} \sum_{j} \int_{\Gamma_{j}}\left\{\mathcal{E}_{j}(t) \frac{\partial G^{(o u t)}\left[\mathbf{R}_{l}, \mathbf{R}_{j}(t)\right]}{\partial N_{j}}\right. \\
\left.-G^{(\text {out })}\left[\mathbf{R}_{l}, \mathbf{R}_{j}(t)\right] \mathcal{F}_{j}(t)\right\} \mathrm{d} t=\mathcal{E}_{l}(t), \\
l=1, \ldots, N, \quad(10 \mathrm{a}) \\
\frac{1}{4 \pi} \int_{\Gamma_{j}}\left\{\mathcal{E}_{j}(t) \frac{\partial G^{(i n)}\left[\mathbf{R}_{l}, \mathbf{R}_{j}(t)\right]}{\partial N_{j}}-G^{(i n)}\left[\mathbf{R}_{l}, \mathbf{R}_{j}(t)\right] \mathcal{F}_{j}(t)\right\} \mathrm{d} t \\
=0, \quad l, j=1, \ldots, N . \quad(10 b)
\end{gathered}
$$

\section{B. Near Field}

In the case of $p$ polarization, once the source functions are known from the set of integral Eqs. (6), the magnetic component of the near field is thus calculated from Eqs. (1a) and $(2 \mathrm{~b})$. To obtain the electric field components from the $y$ component of the magnetic field, we make use of Maxwell's equation:

$$
\nabla \times \mathbf{H}=-i \underset{c}{\omega} \varepsilon \mathbf{E}
$$

Therefore, the electric field has only nonzero components in the $x z$ plane, given outside the scatterers [from Eq. (1a)] by

$$
\begin{aligned}
E_{x}^{(p, \text { out })}(\mathbf{r})= & E_{x}^{(p, i)}(\mathbf{r})-\frac{i c}{4 \pi \omega \varepsilon^{(\text {out })}} \sum_{j} \int_{\Gamma}\left\{\mathcal{H}(t) \frac{\partial^{2} G^{(\text {out })}\left[\mathbf{r}, \mathbf{R}_{j}(t)\right]}{\partial z \partial N_{j}}\right. \\
- & \left.\frac{\partial G^{(\text {out })}}{\partial z}\left(\mathbf{r}, \mathbf{R}_{j}(t)\right) \mathcal{L}_{j}(t)\right\} \mathrm{d} t, \quad(12 \mathrm{a}) \\
E_{z}^{(p, \text { out })}(\mathbf{r})= & E_{z}^{(p, i)}(\mathbf{r})-\frac{E_{y}^{(p, \text { out })}(\mathbf{r})=0,}{4 \pi \omega \varepsilon^{(\text {out })}} \int_{\Gamma}\left\{\mathcal{H}_{j}(t) \frac{\partial^{2} G^{(\text {out })}\left[\mathbf{r}, \mathbf{R}_{j}(t)\right]}{\partial x \partial N_{j}}\right. \\
- & \left.\frac{\partial G^{(\text {out })}\left[\mathbf{r}, \mathbf{R}_{j}(t)\right]}{\partial x} \mathcal{L}_{j}(t)\right\} \mathrm{d} t .
\end{aligned}
$$

Similar expressions are obtained for the near-electricfield components inside the scatterers from Eq. (2b).

When $\mathbf{r}$ is very close to the surface, nonintegrable singularities appear associated with Green's functions derivatives. A simple way to avoid this problem has been pointed out in [30]. The surface electric field is obtained from the surface magnetic field as follows:

$$
\begin{aligned}
& E_{n}^{(p, \text { out })}\left[\mathbf{R}_{j}(t)\right]=\frac{i c}{\gamma \omega \varepsilon^{(\text {out })}} \frac{\mathrm{d} \mathcal{H}_{j}(t)}{\mathrm{d} t}, \\
& E_{t}^{(p, \text { out })}\left[\mathbf{R}_{j}(t)\right]=-\frac{i c}{\gamma \omega \varepsilon^{(\text {out })}} \mathcal{L}_{j}(t),
\end{aligned}
$$

where $E_{n}\left(\mathbf{R}_{j}\right)$ and $E_{t}\left(\mathbf{R}_{j}\right)$ are, respectively, the normal and tangential components of the electric field at the $j$ th surface, and $\gamma=\left[\left(\xi^{\prime}\right)^{2}+\left(\eta^{\prime}\right)^{2}\right]^{1 / 2}$. 
In the case of $s$ polarization, the only nonzero component of the near electric field is directly obtained from Eqs. (7a) and (8b) once the source functions (9) are known from Eqs. (10). If the near/surface magnetic fields were needed, their components could be easily calculated as in Eqs. (12) and (13), but exploiting $\nabla \times \mathbf{E}$ Maxwell's equation instead.

Incidentally, when calculating the near fields close to various scatterers with arbitrary shapes, we incur the numerical problem of determining whether a generic point $\mathbf{r}_{0}$ is located either inside or outside the scatterers. We solve this problem by assuming that a unitary charge is located at the generic point $\mathbf{r}_{0}$. By means of the GaussGreen theorem, we calculate the EM flux across the surface of the scatterer: When the charge is inside the scatterer the flux is unity; otherwise it is zero. Operatively, we calculate the flux of the vector $\hat{\mathbf{r}} /\left|\mathbf{r}-\mathbf{r}_{0}\right|^{2}$ across the surfaces: we obtain nonzero values of the flux only for the inner points.

\section{Far-Field Amplitude and Scattering Cross Section} To calculate the far-field amplitude, we take the asymptotic expression of the Hankel functions for large arguments: $\left|\mathbf{r}-\mathbf{R}_{j}(t)\right| \simeq r-\left[\xi_{j}(t) \sin \theta+\eta_{j}(t) \cos \theta\right]$. In this manner, according to Eqs. (1a) and (7a), we define the $S^{(p, s)}(\theta)$ magnitudes proportional to the scattering amplitudes:

$$
\begin{aligned}
S^{(p)}(\theta)= & \iota\left(\frac{c}{8 \pi \omega \sqrt{\varepsilon^{(\text {out })}}}\right)^{\frac{1}{2}} \times \sum_{j} \int_{\Gamma_{j}}\left\{\imath \frac{\omega}{c} \sqrt{\varepsilon^{(\text {out })}}\right. \\
& \left.\times\left[\eta_{j}^{\prime}(t) \sin \theta-\xi_{j}^{\prime}(t) \cos \theta\right] \mathcal{H}_{j}(t)-\mathcal{L}_{j}(t)\right\} \\
& \times \exp \left\{-\imath \frac{\omega}{c} \sqrt{\varepsilon^{(\text {out })}}\left[\xi_{j}(t) \sin \theta+\eta_{j}(t) \cos \theta\right]\right\} \mathrm{d} t,
\end{aligned}
$$

$$
\begin{aligned}
S^{(s)}(\theta)= & \iota\left(\frac{c \sqrt{\varepsilon^{(\text {out })}}}{8 \pi \omega}\right)^{\frac{1}{2}} \times \sum_{j} \int_{\Gamma_{j}}\left\{l \frac{\omega}{c} \sqrt{\varepsilon^{(\text {out })}}\right. \\
& \left.\times\left[\eta_{j}^{\prime}(t) \sin \theta-\xi_{j}^{\prime}(t) \cos \theta\right] \mathcal{E}_{j}(t)-\mathcal{F}_{j}(t)\right\} \\
& \times \exp \left\{-\imath \frac{\omega}{c} \sqrt{\varepsilon^{(\text {out })}}\left[\xi_{j}(t) \sin \theta+\eta_{j}(t) \cos \theta\right]\right\} \mathrm{d} t .
\end{aligned}
$$

The scattering cross sections (SCS) of the particles can be expressed as

$$
Q_{s c a}^{(p, s)}(\omega)=\int_{0}^{2 \pi} \frac{\left|S^{(p, s)}(\theta \mid \omega)\right|^{2}}{\left|E_{0}^{i}\right|^{2}} \mathrm{~d} \theta
$$

and by means of the optical theorem [23], we obtain the extinction $Q_{\text {ext }}$ and absorption $Q_{a b s}$ cross sections:

$$
Q_{\text {ext }}^{(p, s)}(\omega)=\sqrt{\frac{8 \pi c}{\omega}} \operatorname{Re}\left[S^{(p, s)}\left(\theta=\theta_{\text {forward }}\right)\right],
$$

$$
Q_{a b s}^{(p, s)}(\omega)=Q_{e x t}^{(p, s)}(\omega)-Q_{s c a}^{(p, s)}(\omega)
$$

\section{Numerical Calculations}

We now apply the preceding formulation to calculate SCS and near-field maps associated with metal nanowires of different cross section, either isolated or interacting. In the numerical calculations, the integral equations [see. Eqs. (6) for $p$ and Eqs. (10) for $s$ polarization] are converted into matrix equations by discretizing them through a simple quadrature scheme. The convergence of the resulting surface EM fields [source functions (5) and (9)] with increasing number of sampling points is verified in all cases. The advantage of developing the surface integral scattering equations in parametric form manifests itself for any given cross section profile in that a proper choice of the parametric curves optimizes the minimum number and density of sampling points that ensure numerical convergence.

\section{LIGHT SCATTERING BY SINGLE NANOWIRES}

With the purpose of comparing this method with previous ones, we have calculated the light scattering from a triangular particle as in Kottman et al. [34], where they have solved the problem with a scattering formalism based on the volume integral equation. In Fig. 2, we show the SCS for an isosceles right triangular particle $20 \mathrm{~nm}$ on a side; the amplitude of the incident plane wave is unity, impinging on the top $\left(\theta_{i}=0\right)$. We have rounded off the system corners by $0.25 \mathrm{~nm}$ to provide a more realistic model and to remove numerical instabilities. We observe a complex spectrum with various resonances, very similar to that in Kottman et al. [34], except for the main resonance being slightly higher and blueshifted, probably due to the different numerical approach. The SCS for $s$ polarization is also included for the sake of completeness, revealing as expected no relevant features. As a comment in passing [34], note that a weak peak at $329 \mathrm{~nm}$ is observed in the spectra of the $p$-polarized SCS; this corresponds to a longitudinal mode, stemming from the bulk plasmon resonance when $\mathfrak{R}(\varepsilon) \simeq 0$, its characteristic being fairly constant regardless of the nanowire shape/sizes.

We have studied the near-field amplitude (magnetic and electric fields) at the two significant resonance frequencies of 403 and $358 \mathrm{~nm}$. In Figs. 3(a) and 3(b), we

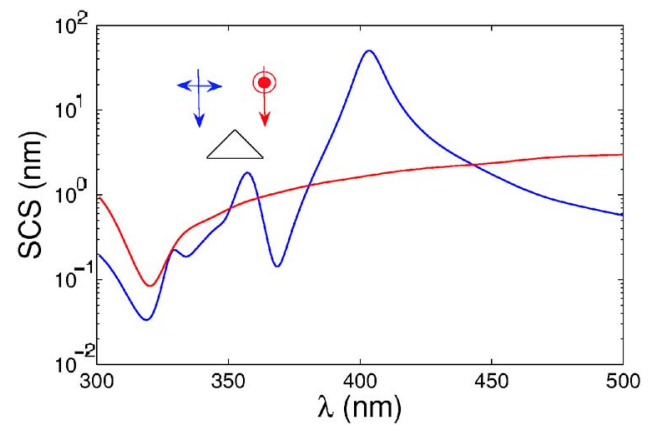

Fig. 2. (Color online) Scattering cross sections for a $20 \mathrm{~nm}$ isosceles right triangle of $\mathrm{Ag}$ for $p$ polarization (blue curve) and for $s$ polarization (red curve). 

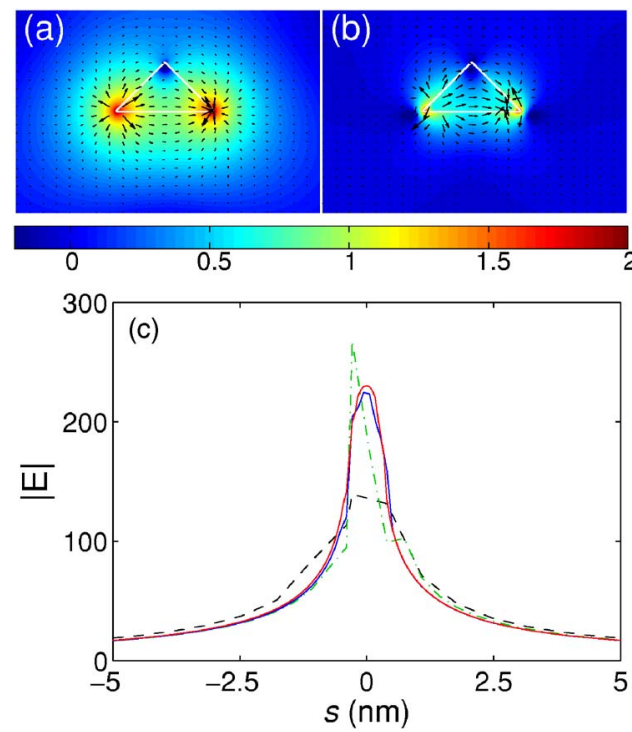

Fig. 3. (Color online) Near-electric-field amplitude distribution in logarithmic scale of light scattering from a $20 \mathrm{~nm}$ isosceles right triangle of $\mathrm{Ag}$ for two plasmon resonances ( $p$ polarization). The amplitude of the incident plane wave is unity and impinges on the top at (a) $\lambda=403 \mathrm{~nm}$, (b) $\lambda=358 \mathrm{~nm}$. (c) Surface electric field amplitude at the base corner of the triangle shown in (a) for different number of sampling points (of the entire triangle perimeter) $N_{p}=100$ (dashed curve), 200 (dotted-dashed curve), 600 (dark solid curve), and 1200 (light solid curve).

show the electric field amplitude distribution inside and outside the nanoparticle in a logarithmic color scale. In both cases, there exists a large field variation between the base corners and the upper corner. While the field amplitude at the two base corners reaches 200 times the incident field amplitude (at the main resonance), it vanishes at the upper corner where a minimum exists.

Incidentally, the surface electric field amplitude at one base corner is explicitly plotted in Fig. 3(c) for increasing number of sampling points, thus revealing the large local field enhancement at the main resonance and also the good convergence of the numerical calculations. The resonances at 403 and $358 \mathrm{~nm}$ present different field distributions at the two base corners. At $403 \mathrm{~nm}$, we obtain a ho-
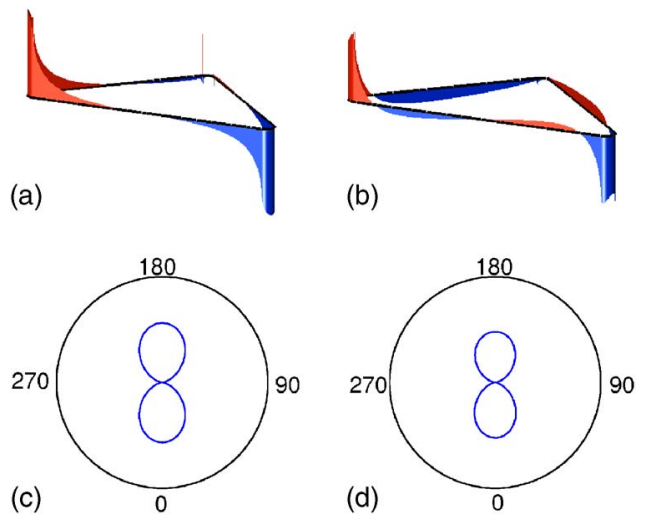

Fig. 4. (Color online) Charge distribution for two plasmon resonances ( $p$ polarization) at (a) $\lambda=403 \mathrm{~nm}$, and at (b) $\lambda=358 \mathrm{~nm}$ (b). These charge distributions correspond to a specific time when the electric field amplitude is maximum at the corners. Far field intensity at (c) $\lambda=403 \mathrm{~nm}$, (d) $\lambda=358 \mathrm{~nm}$. mogeneous, strong field enhancement at the corners. The electric field is mostly normal to the surface in the vicinity of the corners.

The corresponding charge distribution is shown in Fig. 4(a): there is a large charge concentration with opposite sign on each base corner. The normal component has a minimum at the center of the base, where the electric field has only a small tangential component. This resonance is thus a typical dipolar mode, as is confirmed in turn by the angular distribution of scattered intensity in Fig. 4(c).

However, we observe at $358 \mathrm{~nm}$ a nonhomogeneous ( dipolar) behavior near the base corners, with a minimum exactly at the corner and a distance dependence of the near-field amplitude different from that of the resonance at $403 \mathrm{~nm}$ [see Fig. 3(b)]. There is also a large normal electric field component near the base corners at $358 \mathrm{~nm}$, but it nearly vanishes at a point in vacuum very close to the base corners (unlike at $403 \mathrm{~nm}$ ). This is because induced charges of opposite sign [see Fig. 4(b)] are accumulated. The electric field maps shown in Fig. 3 have overall a good agreement with the Kottman et al. [34] results, with the advantages mentioned above that our calculations: (i) scale with the surface; (ii) admit simple, straightforward discretization schemes; and (iii) yield directly the surface fields and charges.

In Fig. 5 we can see that the magnetic field amplitude is smaller than the electric field amplitude with a smooth structure slightly different at each resonance consistent in either case with the charge and electric field distributions mentioned above. For the sake of completeness, the electric near-field intensity maps for $s$ polarization are also included at the wavelengths corresponding to the polarization resonances in Figs. 5(c) and 5(d), showing a fairly structureless pattern with a penetration consistent with the skin depth, as expected.

We have studied in Fig. 6 the SCS and the surface magnetic field intensity for a $20 \times 100 \mathrm{~nm}$ rectangle of silver. To be more realistic, different rounded corners have been taken into account, with circumference of radii of 4,1 , and $0.25 \mathrm{~nm}$. For the sake of comparison, the case of perfect corners is also considered by avoiding the corner points in the numerical implementation, where the position vector derivatives are not defined. It has been demonstrated [32] that the contribution to the far field that is due to the singular points is zero, and the source functions at regular
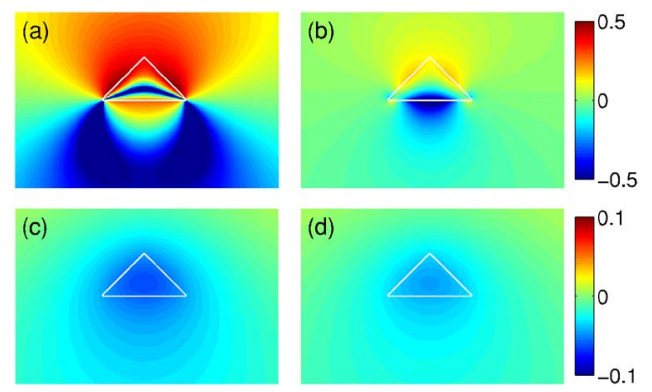

Fig. 5. (Color online) Near-field amplitude distributions in logarithmic scale for a $20 \mathrm{~nm}$ isosceles right triangle of $\mathrm{Ag}$. The amplitude of the incident plane wave is unity and impinges on the top: (a), (b) Magnetic field in $p$ polarization, (c), (d) electric field in $s$ polarization; (a), (c) $\lambda=403 \mathrm{~nm}$; (b), (d) $\lambda=358 \mathrm{~nm}$. 

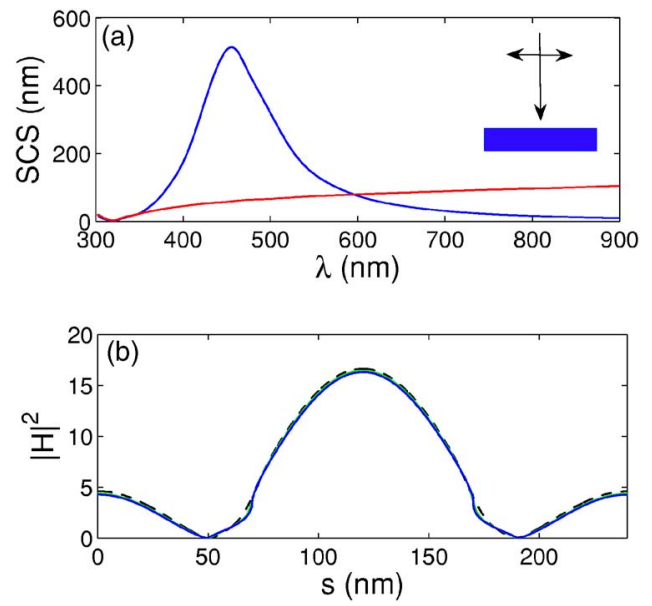

Fig. 6. (Color online) (a) SCS for a Ag rectangular particle with $100 \mathrm{~nm}$ base and $20 \mathrm{~nm}$ height, illuminated on the top in $p$ polarization (blue curve) or in $s$ polarization (red curve). (b) Surface magnetic field intensity for different radius $r$ of the rounded corners: perfect corners (dashed black curve); $r=0.25 \mathrm{~nm}$ (green curve); $r=1 \mathrm{~nm}$ (red curve); $r=4 \mathrm{~nm}$ (blue curve). The $s$ variable is the arc length, with origin at the down half-base, in a clockwise direction.

points are not affected. In fact, our results reveal that the SCS [see Fig. 6(a)] does not vary for all corner radii, exhibiting in this case only one resonance at $\lambda=454 \mathrm{~nm}$. In Fig. 6(b), we show the surface magnetic field intensity for different roundings at the main resonance as a function of the arc length with origin at the half base in a clockwise direction. It is seen that the magnetic field is barely affected by the corner radius, remaining indeed unchanged for radii smaller than $1 \mathrm{~nm}$.

We now explore the surface EM field distribution for the $20 \times 100$ rectangular cross section at the main resonance $[\lambda=454 \mathrm{~nm}$; see Figs. $7(\mathrm{a})$ and $7(\mathrm{~b})]$ plotted in the same manner as the surface magnetic field in Fig. 6(b). The normal component of the electric field shows peaks on the corners, with the same sign and local minimum along each short side [Fig. 7(a)]. The tangential component of the electric field is overall smaller than the normal component [Fig. 7(b)]. Unlike $E_{n}, E_{t}$ has maxima at the centers of the long sides of opposite sign. $E_{t}$ has a jump (ac-
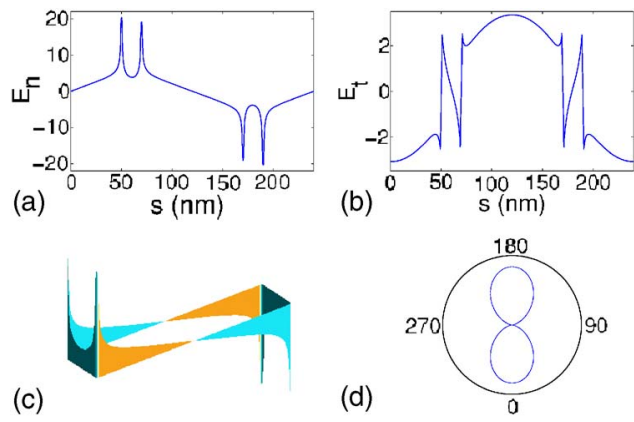

(c)

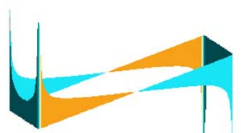

(d)

Fig. 7. (Color online) Surface normal (a) and tangential (b) electric field amplitude for Ag rectangular particle with $100 \mathrm{~nm}$ base and $20 \mathrm{~nm}$ height, illuminated on the top, $s$ variable is the arc length (see Fig. 6). (c) Charge distribution: These field and charge distributions correspond to a specific time when the electric field amplitude is maximum at the corners. (d) Far-field intensity with $\theta=0^{\circ}$ the forward direction, $\lambda=454 \mathrm{~nm}$ (main resonance).

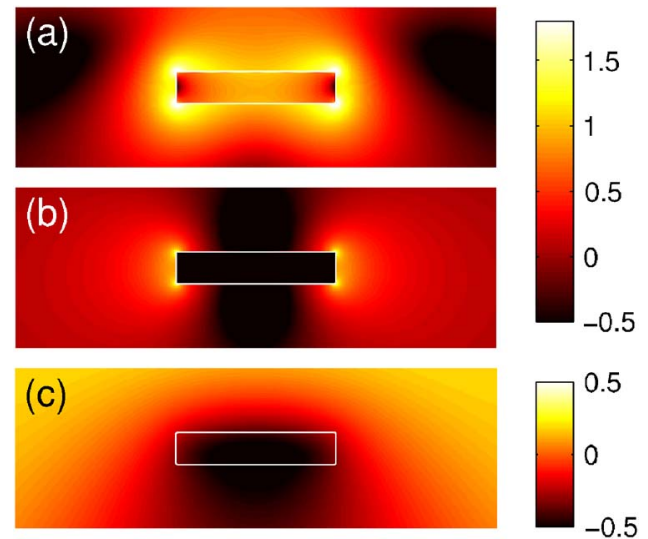

Fig. 8. (Color online) Near-electric-field intensity in $\log _{10}$ scale, normalized to the incident field, for a $\mathrm{Ag}$ rectangular particle with $100 \mathrm{~nm}$ base and $20 \mathrm{~nm}$ height. The plane wave impinges on the top $\left(\theta_{i}=0^{\circ}\right)$ : (a) $\lambda=454 \mathrm{~nm}$ (main resonance, $p$ polarization); (b) $\lambda=900 \mathrm{~nm}$ ( $p$ polarization); (c) $\lambda=454 \mathrm{~nm}$ ( $s$ polarization).

tually a local maximum of the amplitude with a change of sign) on the corners, and at the center of the short sides it is zero.

The surface charge (determined basically by $E_{n}$ ) is concentrated on the corners with the same sign on each short side, being opposite to that on the adjacent long side [see Fig. 7(c)]. This explains as well the jump of the tangential electric component in Fig. 7(b). Such charge distribution can be seen as resembling two parallel horizontal dipoles close to each other, and in turn as a single dipole from the far field, in agreement with the dipolar pattern of the angular distribution of scattered intensity shown in Fig. $7(\mathrm{~d})$.

We show in Fig. 8 the electric near-field intensity maps for $p$ polarization corresponding to the main resonance [Fig. 8(a)] and to $\lambda=900 \mathrm{~nm}$ [off resonance, Fig. 8(b)], along with the electric near-field intensity map for $s$ polarization for $\lambda=454 \mathrm{~nm}$ for comparison [Fig. 8(c)]. At the
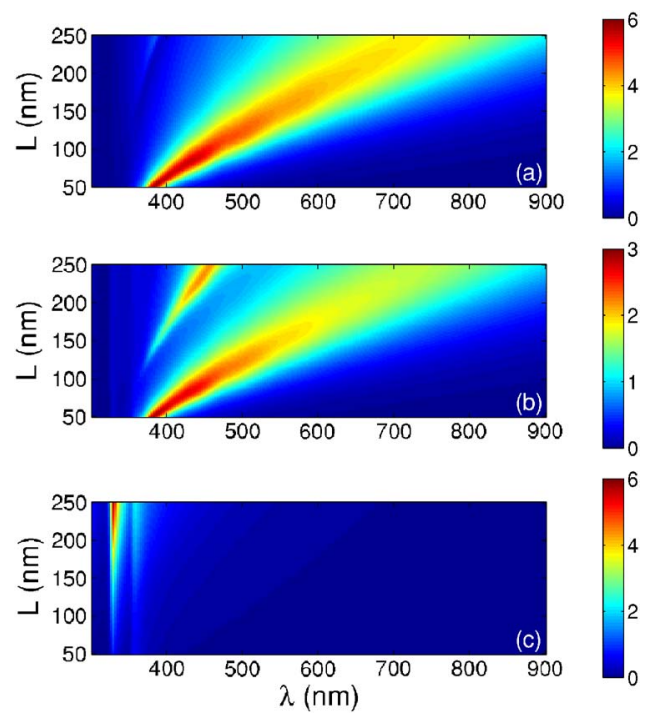

Fig. 9. (Color online) Normalized SCS in $p$ polarization for a Ag rectangular particle with $20 \mathrm{~nm}$ height as a function of the base length $L$ : (a) top incidence $\left(\theta_{i}=0^{\circ}\right)$; (b) oblique incidence $\left(\theta_{i}\right.$ $\left.=45^{\circ}\right)$; (c) lateral incidence $\left(\theta_{i}=90^{\circ}\right)$. 
main resonance, we have a nonzero electric field inside the silver rectangle, mostly parallel to the long side. The electric field intensity is larger at the corners, in agreement with the charge distribution shown in Fig. 7(c). At $\lambda=900 \mathrm{~nm}$, the electric field intensity is larger in a small zone near the corners. In this case, the electromagnetic field does not penetrate into the rectangle (this is not due to the skin depth, which is in fact greater than the height $\sim 22 \mathrm{~nm}$ ); the resulting charge pattern (not shown in Fig. 7) is indeed similar to a dipole. As in the case of the triangle, the near-field pattern in $s$ polarization [Fig. 8(c)] is fairly structureless.

The wavelength of the main resonance in $p$ polarization exhibits a linear dependence on rectangle length in the form $\lambda_{\text {reso }} \propto L / 2$, as revealed in the contour plot of the SCS as a function of length in Fig. 9(a) for light impinging perpendicular to the long side of the rectangle. This is in agreement with a dipolar oscillation of a given charge with dipolar moment directly proportional to the charge separation, as also argued in [11] for rods (for which the linear dependence is slightly different-as $L / 3$-due to the impact of hemispherical ends). In contrast, no qualitative dependence on rectangle length is observed for illumination on the rectangular (fixed) short side [see Fig. 9(c)], which essentially yields, as expected, the main resonance for the dipolar mode across the $20 \mathrm{~nm}$ side, with only a quantitative increase with larger $L$. For oblique incidence, see Fig. 9(b), both resonances are actually excited, the one depending on the long side $L$ being much stronger. A higher-order resonance is also observed for rectangles with $L \gtrsim 150 \mathrm{~nm}$. The corresponding near-field pattern is shown in Fig. 10 for a $20 \times 250 \mathrm{~nm}$, along with the resulting charge distribution. It should be remarked that this resonance is not excited at perpendicular incidence, since the charge symmetry of the corresponding mode is not matched by the electric field oscillation for $\theta_{i}=0^{\circ}$.

In recent works [35], the light scattering properties of nanoparticles of star shape have been experimentally studied. Actually, a star-shaped particle is a paradigmatic example for which parametric coordinates are especially useful. We have carried out calculations for a six-pointed star, the profile of which is simulated as the sum of a circle equation (radius $100 \mathrm{~nm}$ ) and a cosine function with six periods (amplitude $10 \mathrm{~nm}$ ). In Fig. 11(a) the corresponding SCS is shown for an incident field impinging along the upper tip direction. The nanostar spectrum presents three different resonances at $393 \mathrm{~nm}$ (main), $342 \mathrm{~nm}$, and $329 \mathrm{~nm}$ (bulk plasmon). Incidentally, it is worth mentioning that the SCS [Fig. 11(a)] is quite independent of the illumination direction, whereas the surface
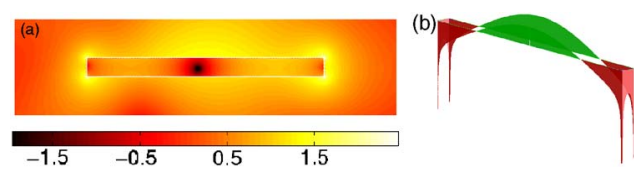

Fig. 10. (Color online) (a) Near-electric-field intensity $\left(\log _{10}\right.$ scale) in $p$ polarization, normalized to the incident field, for a $\mathrm{Ag}$ rectangular particle with $250 \mathrm{~nm}$ base and $20 \mathrm{~nm}$ height. The plane wave impinges on the top $\left(\theta_{i}=45^{\circ}\right)$ at the second-order resonance $\lambda=459 \mathrm{~nm}$. (b) Charge distribution obtained as in Fig. 7(c).

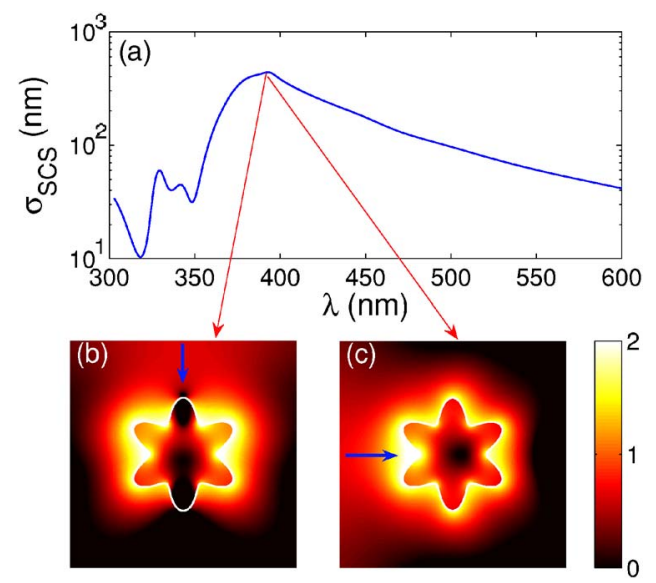

Fig. 11. (Color online) (a) SCS for a Ag six-pointed star with average radius of $100 \mathrm{~nm}$ and oscillation amplitude (see text) of $10 \mathrm{~nm}$ (blue curve). (b), (c) Electric-near-field intensity distribution in $\log _{10}$ scale at $\lambda=393 \mathrm{~nm}$ (main resonance) for two incident directions as denoted by arrows: (b) $\theta_{i}=0^{\circ}$, (c) $\theta_{i}=90^{\circ}$.

EM field might be slightly different. The nanostar main resonance is redshifted with respect to the main resonance of the cylinder of equal radius (not shown here), with a larger SCS. Figures 11(b) and 11(c) show the electric near-field intensity distribution at the main resonance $(\lambda=393 \mathrm{~nm})$ for two incident plane waves impinging on the top $\left(\theta_{i}=0^{\circ}\right)$ and on the left $\left(\theta_{i}=90^{\circ}\right)$, respectively. We can see that the field intensity is symmetric with respect to the incident direction. For $\theta_{i}=0$, the electric field intensity has minima at the upper and lower tips. This behavior is due to tangential charge variations being forbidden on those tips, as imposed by the incident polarization; it corresponds to a nonfavorable charge distribution, as shown in [36] for Gaussian ridges on a flat metal surface.

The same argument applies to the electric field minima on the upper corner in the triangle above. In contrast, the tangential electric intensity presents maxima on all valleys for both incident directions, along with large normal electric field intensity at the adjacent walls, where charge with opposite sign is accumulated, as seen in Fig. 11(c) [and also in Fig. 11(b) albeit weaker at the walls of the upper and lower tips]. Basically, the near-field pattern in the valleys has dipolelike behavior [Fig. 11(c)] due to such opposite charge concentration on either dip wall [36]. Recall that field enhancements of more than two orders of magnitude are found between star tips; nanostar dips could thus play the role of hot spots for SERS applications and related ones. Overall, there exists a complex multipolar charge distribution at the main resonance, related to the star-shape symmetry, that leads to a complex far-field pattern; this will be investigated in detail elsewhere.

\section{LIGHT SCATTERING BY INTERACTING NANOWIRES}

We now show that the surface integral equation formulation can also be exploited to investigate the light scattering from strongly interacting nanowires in various cases exhibiting coupled plasmon resonances. First, for the sake of comparison, we present in Fig. 12 a study of the plas- 

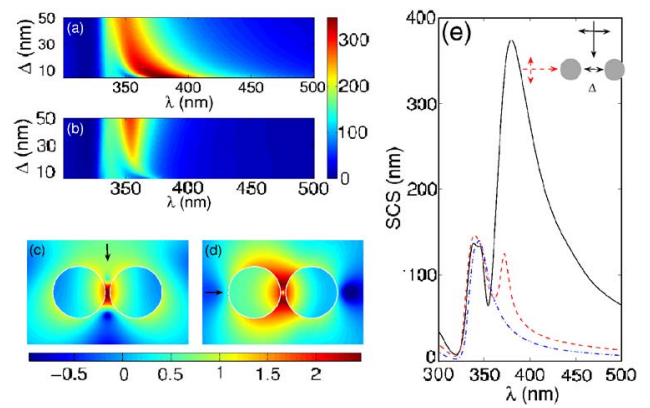

Fig. 12. (Color online) (a), (b) SCS for two Ag cylinders ( $p$ polarization) with $25 \mathrm{~nm}$ radius for different separation, illuminated on the top (a) or on the left (b). (c), (d) Near-electric-field intensity at the main resonances for a separation of $5 \mathrm{~nm}\left(\log _{10}\right.$ scale normalized to the incident field, $p$ polarization): (c) $\lambda=380 \mathrm{~nm}$; (d) $\lambda=372 \mathrm{~nm}$ ( $p$ polarization), illumination direction as indicated. (e) SCS for a separation of $5 \mathrm{~nm}$, with illumination (see inset) on the top (black solid curve), on the left (red dashed curve), and SCS for only one cylinder (blue dotted-dashed curve).

monic resonances for two $\mathrm{Ag}$ cylinders of $25 \mathrm{~nm}$ radius as the distance $\Delta$ between them is reduced, as in [14]. For large enough $\Delta$, we retrieve the SCS of two separated, noninteracting cylinders, as expected. We can see that a strong coupling effect exists for small distances, where a splitting of the plasmon resonance takes place. This effect is more obvious for incidence normal to the dimer axis; see Fig. 12(a). The stronger plasmon resonance (at $\approx 380 \mathrm{~nm}$ for $\Delta \approx 5 \mathrm{~nm}$ ) corresponds to a large charge concentration of opposite sign between the cylinders. This resonance is redshifted with respect to the isolated cylinder mode, because the interaction energy between opposite charges is smaller for small distances.

The resulting near-field intensity map is shown in Fig. 12(c), which indeed reveals the strong field enhancement and localization within the dimer gap directed along the dimer axis. The second, symmetric, weaker resonance (at $\approx 340 \mathrm{~nm}$ for $\Delta \approx 5 \mathrm{~nm}$ ) is present only for small distances; it corresponds to the dipolar mode of an isolated cylinder with the same charge across the dimer gap (thus not shown in Fig. 12). On the other hand, when the direction of the incident field is parallel to the dimer axis (see Fig. 12(e)), two resonances are also found, but with substantially smaller SCS. In this case, the SCS splitting for smaller distances is accompanied by a decrease in strength, because the charge distribution of each cylinder oscillates with opposite phase with respect to the other cylinder $[10,14,37]$. The near-field pattern [see Fig. 12(d)] shows also a large field enhancement between both cylinders, though weaker and broader than that of the resonance in Fig. 12(c) and indeed directed perpendicular to the dimer axis (with a minimum at the center).

In Fig. 13(a) we show the SCS of a Ag rectangular dimer as a function of the gap width $\Delta$. The dimensions of each rectangle are $20 \times 100 \mathrm{~nm}^{2}$. Similar rectangular dimers have been termed nanoantennas $[4,5]$ for their ability to enhance light coupling and emission. When the two rectangles are well apart, the SCS resembles that of a single rectangle (nearly twice as intense), with the main resonance located at the same frequency [see Fig. 9(a)]. On reducing the gap width, the two rectangles begin to couple, so that the main resonance is redshifted as a con-

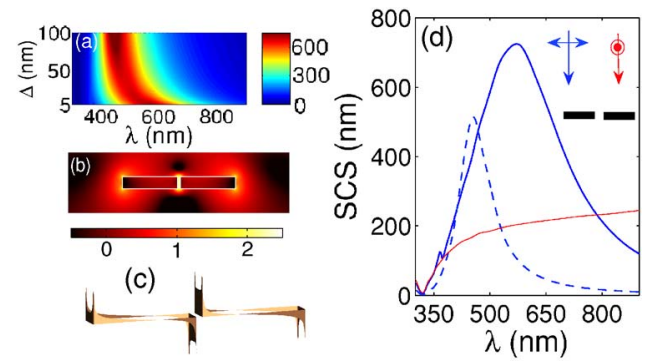

Fig. 13. (Color online) (a) SCS for a Ag rectangular dimer (100 nm base and $20 \mathrm{~nm}$ height) as a function of the gap width $\Delta$, for $p$-polarized incidence at $\theta_{i}=0^{\circ}$ (perpendicular to the dimer axis). (b) Near-electric-field intensity ( $\log _{10}$ scale) normalized to the incident field, for a $p$-polarized plane wave impinging on the top at $\lambda=573 \mathrm{~nm}$ (main resonance). (c) Charge distribution on the surface of the rectangle dimer as in (b). (d) SCS for a Ag rectangular dimer with $100 \mathrm{~nm}$ base, $20 \mathrm{~nm}$ height, and $5 \mathrm{~nm}$ gap, illuminated on the top in $p$ polarization (blue solid curve) and in $s$ polarization (red curve). The SCS for a single rectangle ( $p$ polarization) is also shown (blue dashed curve).

sequence of the charge accumulation across the gap [11]. In the limit of touching rectangles, the SCS should coincide with that of a single rectangle with length $2 L$ $=200 \mathrm{~nm}$, with a main resonance at $\approx 620 \mathrm{~nm}$ [see Fig. 9 (a)]; this wavelength is in turn the upper limit for the coupling-induced redshift of the main resonance in Fig. 13(a). The near-field distribution for $\Delta=5 \mathrm{~nm}$ at resonance $(\lambda=573 \mathrm{~nm})$ in Fig. 13(b) illustrates the strong coupling regime; the corresponding SCS is explicitly plotted in Fig. 13(d), along with that for a single rectangle. In Fig. 13(b) it is shown that, as a consequence of such strong coupling, differences arise in Fig. 13 at each rectangle with regard to the electric near-field intensity for the single rectangle resonance [see Fig. 8(a)] in the form of asymmetry of the charge distribution inside each $\mathrm{Ag}$ rectangle of the dimer. The dimer charge is clearly concentrated in the gap (short side), with opposite signs, being weaker at the two extremes of the dimer. The charge node in the middle of the long sides is shifted toward the gap when the latter is decreased. The electric field is larger between the nanowires and predominantly directed along the dimer axis; very large field enhancements, of nearly three orders of magnitude, are found. Similar field enhancements have been exploited to strongly drive other emission processes (two-photon photoluminescence, fluorescence, white-light continuum, etc.), both quantitative and qualitatively, playing the role of optical nanoantennas $[4,5]$.

\section{CONCLUSIONS}

In this work we have developed an implementation in parametric coordinates of the light scattering formulation based on Green's theorem surface integral equations, thus far used only for single-valued interfaces and/or simple close contours, in order to account for an arbitrary number of wires (or 2D particles) of arbitrary shape and dielectric function. This formulation is formally exact and has the decisive advantage that the numerical implementation scales with the particle surface rather than with its volume. 
A variety of metallic nanowires were investigated exploring the rich phenomenology associated with plasmon resonances. Two configurations (triangles and two cylinders) already investigated in the literature were reproduced and extended to asses the validity and versatility of the formulation. Then complex geometries of interest in surface-plasmon- and nano-optics were studied, either for isolated or interacting nanowires, for which the impact of either shape or coupling on the plasmon particle resonances is crucial, including spectra of scattering cross sections and near-field intensity maps on/off resonance. Such shapes as circles, triangles, stars, and thin rectangles, forming dimers (nanoantennas) were studied.

\section{ACKNOWLEDGMENTS}

This work was supported in part by the Ministerio de Educación y Ciencia of Spain (Grants FIS2006-07894 and FIS2004-0108) and the Comunidad de Madrid (Grant MICROSERES S-0505/TIC-0191 and V.G.'s scholarship).

\section{REFERENCES}

1. S. A. Maier and H. A. Atwater, "Plasmonics: Localization and guiding of electromagnetic energy in metal/dielectric structures,” J. Appl. Phys. 98, 011101 (2005).

2. E. Ozbay, "Plasmonics: Merging photonics and electronics at nanoscale dimensions," Science 311, 189-193 (2006).

3. N. Fang, H. Lee, C. Sun, and X. Zhang, "Sub-diffractionlimited optical imaging with a silver superlens," Science 308, 534-537 (2005).

4. P. Mühlschlegel, H. J. Eisler, O. J. F. Martin, B. Hecht, and D. W. Pohl, "Resonant optical antennas," Science 308, 1607-1609 (2005).

5. J. N. Farahani, D. W. Pohl, H. J. Eisler, and B. Hecht, "Single quantum dot coupled to a scanning optical antenna: A tunable superemitter," Phys. Rev. Lett. 95, 017402 (2005).

6. T. Kalkbrenner, U. Håkanson, A. Schädle, S. Burger, C. Hankel, and V. Sandoghdar, "Optical microscopy via spectral modifications of a nanoantenna," Phys. Rev. Lett. 95, 200801 (2005).

7. A. Ono, J. Kato, and S. Kawata, "Subwavelength optical imaging through a metallic nanorod array," Phys. Rev. Lett. 95, 267407 (2005).

8. A. Madrazo and M. Nieto-Vesperinas, "Scattering of electromagnetic waves from a cylinder in front of a conducting plane," J. Opt. Soc. Am. A 12, 1268-1309 (1995).

9. J. P. Kottmann, O. J. F. Martin, D. R. Smith, and S. Schultz, "Plasmon resonances of silver nanowires with a nonregular cross section," Phys. Rev. B 64, 235402 (2001).

10. W. Rechberger, A. Hohenau, A. Leitner, J. R. Krenn, B. Lamprecht, and F. R. Aussenegg, "Optical properties of two interacting gold nanoparticles," Opt. Commun. 220, 137-141 (2003).

11. J. Aizpurua, G. W. Bryant, L. J. Richter, F. J. García de Abajo, B. K. Kelley, and T. Mallouk, "Optical properties of coupled metallic nanorods for field-enhanced spectroscopy," Phys. Rev. B 71, 235420 (2005).

12. K. L. Shuford, M. A. Ratner, and G. C. Schatz, "Multipolar excitation in triangular nanoprisms," J. Chem. Phys. 123, 114713 (2005).

13. U. Hohenester and J. Krenn, "Surface plasmon resonances of a single and coupled metallic nanoparticles: A boundary integral method approach," Phys. Rev. B 72, 195429 (2005).

14. I. Romero, J. Aizpurua, G. W. Bryant, and F. J. García de Abajo, "Plasmons in nearly touching metallic nanoparticles: singular response in the limit of touching dimers," Opt. Express 14, 9988-9999 (2006).

15. F. Moreno, F. González, and J. M. Saiz, "Plasmon spectroscopy of metallic nanoparticles above flat dielectric substrates," Opt. Lett. 31, 1902-1904 (2006).

16. S. E. Sburlan, L. A. Blanco, and M. Nieto-Vesperinas,
"Plasmon excitation in sets of nanoscale cylinders and spheres," Phys. Rev. B 73, 035403 (2006).

17. V. Giannini, J. A. Sánchez-Gil, J. V. García-Ramos, and E. R. Méndez, "Collective model for the surface-plasmonmediated electromagnetic emission from molecular layers on metallic nanostructures," Phys. Rev. B 75, 235447 (2007).

18. E. J. Zeman and G. C. Schatz, "An accurate electromagnetic theory study of surface enhancement factors for $\mathrm{Ag}, \mathrm{Au}, \mathrm{Cu}, \mathrm{Li}, \mathrm{Na}, \mathrm{Al}, \mathrm{Ga}, \mathrm{In}, \mathrm{Zn}$, and $\mathrm{Cd}$," J. Phys. C 91, 634-643 (1987).

19. J. A. Sánchez-Gil and J. V. García-Ramos, "Local and average electromagnetic enhancement in surface-enhanced Raman scattering from self-affine fractal metal substrates with nanoscale irregularities," Chem. Phys. Lett. 367, 361-366 (2003).

20. H. Xu, J. Aizpurua, M. Käll, and P. Apell, "Electromagnetic contributions to single-molecule sensitivity in surfaceenhanced Raman scattering," Phys. Rev. E 62, 4318-4324 (2000).

21. S. Nie and S. R. Emory, "Probing single molecules and single nanoparticles by surface-enhanced Raman scattering," Science 275, 1102-1106 (1997).

22. K. Kneipp, Y. Wang, H. Kneipp, L. T. Perlman, I. Itzkan, R. R. Dasari, and M. S. Feld, "Single molecule detection using surface-enhanced Raman scattering (SERS)," Phys. Rev. Lett. 78, 1667-1670 (1997).

23. C. F. Bohren and D. R. Huffman, Absorption and Scattering of Light by Small Particles (Wiley, 1998).

24. A. Hohenau, J. R. Krenn, J. Beermann, S. I. Bozhevolnyi, S. G. Rodrigo, L. Martin-Moreno, and F. J. Garcia-Vidal, "Spectroscopy and nonlinear microscopy of Au nanoparticle arrays: experiment and theory," Phys. Rev. B 73, 155404 (2006).

25. C. Girard and A. Dereux, "Near-field optics theories," Rep. Prog. Phys. 59, 657-699 (1996).

26. A. A. Maradudin, T. Michel, A. R. McGurn, and E. R. Mendéz, "Enhanced backscattering of light from a random grating," Ann. Phys. (N.Y.) 203, 255-307 (1990).

27. J. A. Sánchez-Gil and M. Nieto-Vesperinas, "Light scattering from random rough dielectric surfaces," J. Opt. Soc. Am. A 8, 1270-1286 (1991).

28. F. J. García de Abajo and J. Aizpurua, "Numerical simulation of electron energy loss near inhomogeneous dielectrics," Phys. Rev. B 56, 15873-15884 (1997).

29. M. Nieto-Vesperinas, Scattering and Diffraction in Physical Optics (Wiley, 1991)

30. J. A. Sánchez-Gil, J. V. García-Ramos, and E. R. Méndez, "Near-field electromagnetic wave scattering from random self-affine fractal metal surfaces: Spectral dependence of local field enhancement and their statistics in connection with surface-enhanced Raman scattering," Phys. Rev. B 62, 10515-10525 (2000)

31. P. B. Johnson and R. W. Christy, "Optical constants of the noble metals," Phys. Rev. B 6, 4370-4379 (1972).

32. A. Mendoza-Suárez and E. R. Méndez, "Light scattering by a reentrant fractal surface," Appl. Opt. 36, 3521-3531 (1997).

33. C. I. Valencia, E. R. Méndez, and B. Mendoza, "Secondharmonic generation in the scattering of light by twodimensional particles," J. Opt. Soc. Am. B 20, 2150-2161 (2003).

34. J. P. Kottmann, O. J. F. Martin, D. R. Smith, and S. Schultz, "Spectral resonances of plasmon resonant nanoparticles with a non-regular shape," Opt. Express 6, 213-219 (2001).

35. C. L. Nehl, H. Liao, and H. Hafner, "Optical properties of star-shaped gold nanoparticles," Nano Lett. 6, 683-688 (2006).

36. J. A. Sánchez-Gil, "Localized surface-plasmon polaritons in disordered nanostructured metal surfaces: Shape versus Anderson-localized resonances," Phys. Rev. B 68, 113410 (2003).

37. J. P. Kottmann and O. J. F. Martin, "Plasmon resonant coupling in metallic nanowires," Opt. Express 8, 665-663 (2001). 